\title{
Assessment of vitamin A supplementation coverage and its association with childhood illness in Boloso Sore Woreda, Welayta Zone, SNNP Region, Ethiopia
}

\author{
Samson Gebremedhin ${ }^{1}$, Eskinder Loha ${ }^{2}$, Yewelsew Abebe ${ }^{1}$, Gulelat Dese ${ }^{3}$
}

\begin{abstract}
Background: Vitamin A plays vital role in the physiology of vision and immunity. Globally quarters of a billion children are Vitamin A deficient. Vitamin A supplementation of children and mothers during postpartum period is a key strategy to avert the deficiency. However the effect of Vitamin A supplementation on incidence of childhood illnesses is controversial.

Objective: To assess the effect of Vitamin A supplementation on the reduction of common childhood illnesses, and to measure the coverage of vitamin A supplementation among children aged 6-59 months and mothers during postpartum period.

Methods: A retrospective cohort study which involves comparison between Vitamin A supplemented and nonsupplemented children was conducted in Feb 2008 in Boloso Sore Woreda, Wolayta Zone, SNNPR. A total of 1601 children aged 6-59 months were selected using Probability Proportional to Size (PPS) sampling technique.

Results: Vitamin A supplementation coverage among children (6-59 months) was 83.1\% and among women (in postpartum period) who had given birth in the preceding 12 months of the survey was $13.2 \%$. In comparison with the corresponding level in 2005, the coverage in the area has increased significantly $(\mathrm{P}<0.05)$. Most of the vitamin $\mathrm{A}$ supplementation coverage was achieved through Enhanced Outreach Strategy. Vitamin A supplementation status was not significantly associated with history of Fever [AOR=1.26 (95\%CI 0.89-1.77)], Cough or rapid breathing/difficulty in breathing $[\mathrm{AOR}=1.15(95 \% \mathrm{CI} 0.77-1.72)]$, Eye infection $[\mathrm{AOR}=1.22(95 \% \mathrm{CI}$ 0.78-1.89)], and Diarrhea $[\mathrm{AOR}=0.98$ (95\%CI 0.64-1.52)].
\end{abstract}

Recommendations: The effect of Vitamin A supplementation on reduction of childhood illnesses should be assessed through longitudinal studies among Vitamin A deficient children. [Ethiop. J. Health Dev. 2009;23(3):223-228]

\section{Introduction}

Vitamin A and its metabolites play diverse roles in physiology, ranging from incorporation into vision pigments to controlling transcription of important genes (1). Vitamin A is commonly known as the anti-infective vitamin, because it is required for normal functioning of the immune system. Retinol and its metabolites are required to maintain the integrity and function of the skin and mucosal cells which function as a barrier and form the body's first-line of defense against infection (2).

Vitamin A deficiency (VAD) remains a serious public health problem in developing countries. Pre-school aged children and women during pregnancy or lactation tend to suffer the most widespread and severe effect of VAD (3). Other segments of a population can also be vulnerable to the deficiency as a result of consumption of Vitamin A deficient staple diet or lack of access to Vitamin A rich foods due to climatic, seasonal and economical factors (4).

Currently, about 7.2 million women in the developing countries are Vitamin A deficient and another 13.6 million have low Vitamin A status. Globally, 140-250 million children under five years of age are affected by vitamin A deficiency. These children suffer increased risk of death, blindness and illness (3). The UN Special
Session on Children in 2002 had set the goal of eliminating vitamin A deficiency and its consequences by the year 2010. Especially in areas where the intake of vitamin $\mathrm{A}$ is inadequate, provision of the vitamin through a combination of breastfeeding, dietary improvement, food fortification, and supplementation is recommended. Such interventions should also be augmented by nutrition education (5).

Several studies over the years have established that vitamin A deficiency is a major public health problem in Ethiopia. Studies conducted starting from late 1950's consistently indicated that the prevalence of Bitot's spot among preschool children is well above the cutoff point of $0.5 \%$ which indicates its public health significance (68). Recent studies reported the same (9-12). According to a recent $\mathrm{WHO}$ estimate, the national prevalence of vitamin A deficiency among preschool children in Ethiopia is $61.2 \%$ which is among the highest in SubSaharan Africa (3).

Since 1987, WHO has advocated the routine administration of vitamin A supplement for children aged 6-59 months every four to six months and mothers soon after delivery. This is considered as an inexpensive, quick, and effective way to improve vitamin A status in areas where vitamin A deficiency is a public health

${ }^{1}$ Hawassa University, College of Agriculture, Tel. +251916822815, E-mail samsongmgs@,hayoo.com;

${ }^{2}$ Hawassa University, College of Health Sciences; ${ }^{3}$ Addis Ababa University, Faculty of Sciences 
problem (5). However, in Ethiopia the coverage of vitamin A supplementation among preschool children and women in the postpartum period is below satisfactory level. According to Demographic and Health Survey (DHS) Ethiopia 2005, among preschool children aged 12-59 months, only $45.8 \%$ received the supplement in the preceding 6 months of the survey and among women in the postpartum period, only $20.6 \%$ received the supplement in the first two months after their delivery (13).

The effect of Vitamin A supplementation on incidence of childhood illnesses is controversial. The Beaton report concluded that all-cause mortality among children aged 6-59 months can be reduced by $23 \%$ through vitamin A supplementation $(5,14)$. Similar findings have also been reported by Glasziou P (15), Fawiz W (16), Victor M et al. (17) and George $H$ et al. (18). In Ethiopia, an interventional study conducted in 1997 by Haidar et al. concluded that Vitamin A supplementation significantly reduces incidence of fever, diarrhea, measles, conjunctivitis, stunting and wasting among children aged 6-72 months (19).

However, a number of studies have also reported that there is no significant association between Vitamin A supplementation and incidence of common childhood illnesses like Acute Respiratory Infection (ARI) and Diarrheal Diseases. This includes the findings of Biswas R et al. (20), Brown $\mathrm{N}$ and Roberts C (21), Abdelijaber $\mathrm{M}$ et al. (22) and Humphrey $\mathrm{J}$ et al. (23). Even few studies have reported supplementation of vitamin A increases incidence of childhood illness. Stansfield S reported children supplemented with Vitamin A have higher risk of Diarrhea, Rhinitis and Cough (24). Similarly Michael J et al. witnessed high dose vitamin A supplements increased the incidence of acute respiratory illnesses (25).

Hence, this study was conducted to assess the effect of Vitamin A supplementation on the reduction of common childhood illnesses among children aged 6-59 months and to measure the coverage of vitamin $A$ supplementation in same age of children and women who had given birth in the preceding 1 year of the survey.

\section{Methods}

A retrospective cohort, quantitative study which involves comparison between Vitamin A supplemented and nonsupplemented children was conducted in the former Boloso Sore Woreda, Wolayta Zone, SNNPR. The study was carried out in Feb 2008.

The former Boloso Sore Woreda, (recently divided into Boloso Sore, Damot Sore and Bolosso Bombe Woredas and Areka town administration) is predominantly inhabited by the Welayta people. According to Central Statistical Authority 2005 report, the woreda had an estimated total population of 316,699 with male to female ratio of $1: 1.04$. Nine percent of the population is urban dweller. With an estimated area of $632.7 \mathrm{Km}^{2}$, the woreda had an estimated population density of 567 people $/ \mathrm{Km}^{2}$ (26). In Welayta zone where the study area is located, the primary school enrollment rate is $78 \%$ and primary health coverage does not exceed $53 \%$. The zone is also identified as a chronically food insecured area (27).

The data for this article were extracted from a dataset of large scale survey conducted by the authors as evaluation of ESHE's (Essential Services for Health in Ethiopia) health and nutrition sector interventions in Bolosso Sore Woreda. In the survey, Probability Proportional to Size (PPS) sampling technique was used to select representative households. Initially all the sub-kebeles in the aforementioned study area were listed. With the intention of sampling 80 clusters, the total population of 316,699 was divided by 80 to give sampling interval of 3959. A number between 0-3959 was selected randomly as starting point and 80 clusters were selected. From each cluster, 30 households were selected by "Random walk" method.

The procedure of the random walk method was as follows. First the geographic center of the sub-kebele was located and a random direction was selected by spinning a pen or pencil. Then the first five households in the selected directions were skipped and starting from the $6^{\text {th }}$ house every other household was included into the study. At times when the data collectors reached the border of the sub-kebele, they moved in a clockwise fashion until they got 30 households. In such manner, 2400 households were included in the main study. However for this specific article, the data of 1601 children aged 6-59 months (from 1270 households having one or more children aged 6-59 months) were used.

The data were collected using 18 data collectors and 6 supervisors. All the data collectors and supervisors were at least diploma holders in the field of Health Sciences. They also had long-term experience of working in the study area. Prior to data collection 5 days of intensive training, including field practice, was offered to data collectors. The questionnaire was pre-tested and corrected accordingly.

Information was collected on wide range of variables including socio-demographic and economic information of sampled households and children aged 6-59 months, the Vitamin A supplementation history of each child in the preceding 6 months of the survey and illness history of each child in the preceding 2 weeks of the survey.

Before analysis, the adequacy of the sample size was assessed. In order to estimate the coverage of Vitamin A supplementation, using single proportion sample size calculation formula (with 95\% CI, 5\% margin of error and expected Vitamin A supplementation coverage of $50 \%$ ), sample size of 384 would suffice. The adequacy 
was also checked to satisfy the minimum sample size requirement for applying Logistic Regression (having at least 20 events per parameter in the model for every class of the dependent variable). Accordingly the sample size of 1601 children was judged to be adequate for the analysis.

The data were entered and analyzed using SPSS 15.0 for windows. Comparison of two proportions for independent samples was carried out using MStat statistical software. Prior to the analysis, the whole data were cleaned. The data analysis was done using frequencies, percentages, crude and adjusted odds ratio etc. $P$ value of 0.05 was considered as level of significance. After verifying that all the assumptions of logistic regression are met, multinomial logistic regression was used to control cofounders and to verify association between the dependant (history of illness) and independent (Vitamin A supplementation history) variables.

The study was conducted in confirmation with national and international research ethics principles. Prior to data collection, permission was taken from regional health bureau. In addition informed consent was taken from every participant. To ascertain confidentiality, data were collected and recorded in anonymous manner. Administrative bodies of the area were informed about the aim and scope of the study and permission was also gained from them.

\section{Operational Definitions}

Eye infection: The occurrence of purulent discharge and redness of eye in the preceding 2 weeks of the survey as reported by the mother.

Illness: The occurrence of one or more symptoms of illness in the preceding 2 weeks of the survey as reported by the mother.

Vitamin A supplemented child: A child who was given Vitamin A capsule in the preceding 6 months of the survey as reported by the mother (after the mother is shown the capsule).

\section{Results \\ Information on Socio-demographic Information of Children and Households}

From 1270 households, a total of 1601 children aged 659 months were identified and included to the study. The data of $4(0.2 \%)$ children were excluded from analysis since their Vitamin A supplementation history can not be ascertained. Hence analysis was done with the remaining 1597 subjects. Just more than half, 831 (52.0\%), of the children were boys. Age wise 251 (15.7\%), 314 (19.7\%) and 1032 (64.7\%) were in 6-11 mo, 12-23 mo and 24-59 months age categories, respectively (Table 1).

Table 1: Household characteristics of respondents, Bolosso Sore Woreda, Feb. 2008.

\begin{tabular}{lcc}
\hline & $\begin{array}{c}\text { Frequency } \\
\text { (n=1270 households) }\end{array}$ & Percent \\
\hline Educational status of mothers & 843 & 66.4 \\
Illiterate & 7 & 0.6 \\
Informal education & 282 & 22.2 \\
Primary (1-6) & 124 & 9.8 \\
Secondary (7-10) & 14 & 1.1 \\
Higher & & 25.7 \\
Household size & 326 & 65.0 \\
1-4 & 826 & 9.3 \\
5-8 & 118 & \\
> 8 & & 53.5 \\
Access to the nearest health facility & 680 & 24.7 \\
(one-way estimated walking distance) & 314 & 18.0 \\
< 30 minutes & 228 & 3.4 \\
30 minutes to 1 hr & 43 & 0.4 \\
1 to 2 hrs & 5 & 50.2 \\
2 to 3 hrs & & 25.5 \\
> 3 hrs & 638 & 10.0 \\
Main source of drinking water & 324 & 8.3 \\
Public standpipe & 127 & 4.5 \\
Unprotected dug well & 106 & 2.3 \\
Unprotected spring & 57 & 85.0 \\
Protected spring & 17 & 14.3 \\
Protected dug well & & 0.6 \\
Household connection & 1080 & 83.1 \\
Ownership and type of latrine & 182 & 16.9 \\
Pit latrine & 8 & \\
Open field disposal & 1055 & \\
Other types of latrines & 215 & \\
Ownership of at least one bed net & & \\
Yes & & \\
No & & \\
\hline
\end{tabular}


Vitamin A Supplementation Coverage: All mothers were shown a Vitamin A capsule and asked whether their child has taken a similar one in the preceding 6 months of the survey. Of 1597 children aged 6-59 months, 1327 (83.1\%) had received Vitamin A supplement in the reference period. Majority of the children who got vitamin A supplementation received the service through
Enhanced Outreach Strategy (EOS) campaigns and routine immunization programs. Insignificant number of children got the service during well and sick child visit of health institutions (Table 2). Of 469 women who had given birth in the preceding 12 months of the survey, only $13.2 \%$ had received Vitamin A supplement within 45 days after their recent delivery.

Table 2: Vitamin A Supplementation among Children Age 6-59 months,

Bolosso Sore Woreda, Feb 2008.

\begin{tabular}{lcc}
\hline Modality of Vitamin A delivery & $\begin{array}{c}\text { Frequency } \\
(\mathbf{n}=\mathbf{1 3 2 7})\end{array}$ & Percent \\
\hline EOS Campaign & 809 & 61.0 \\
Routine immunization & 446 & 33.6 \\
Home visit & 26 & 2.0 \\
Growth monitoring & 15 & 1.1 \\
Sick child visit of health institutions & 13 & 1.0 \\
Others & 18 & 1.4 \\
\hline
\end{tabular}

Effect of Vitamin A Supplementation on Reduction of Childhood Illnesses

With the intension of assessing the effect of Vitamin A supplementation on reduction of childhood illness, mothers were asked whether their children (age 6-59 months) were ill in the past two weeks of the survey or not. Accordingly, 628 (39.3\%) children had one or more symptoms of illness in the reference period. The leading reported illness/symptoms were fever, cough or rapid breathing/difficulty in breathing, eye infection and diarrhea (Table 3).

\begin{tabular}{|c|c|c|c|c|c|c|}
\hline \multirow{2}{*}{ IIIness/Ailments } & \multicolumn{2}{|c|}{$\begin{array}{l}\text { Children who received } \\
\text { Vitamin A supplement }\end{array}$} & \multicolumn{2}{|c|}{$\begin{array}{c}\text { Children who didn't receive } \\
\text { Vitamin A supplement }\end{array}$} & \multicolumn{2}{|c|}{ Total } \\
\hline & $\begin{array}{c}\text { Frequency } \\
(n=1327)\end{array}$ & Percent & $\begin{array}{c}\text { Frequency } \\
(\mathrm{n}=270)\end{array}$ & Percent & $\begin{array}{c}\text { Frequency } \\
(n=1597)\end{array}$ & Percent \\
\hline Fever & 276 & 20.8 & 53 & 19.6 & 329 & 20.6 \\
\hline $\begin{array}{l}\text { Cough or rapid/difficulty in } \\
\text { breathing }\end{array}$ & 170 & 12.8 & 36 & 13.3 & 206 & 12.9 \\
\hline Eye infection & 145 & 10.9 & 29 & 10.7 & 174 & 10.9 \\
\hline Diarrhea & 131 & 9.9 & 31 & 11.5 & 162 & 10.1 \\
\hline Tonsillitis & 50 & 3.8 & 7 & 2.6 & 57 & 3.6 \\
\hline Ear infection & 21 & 1.6 & 9 & 3.3 & 30 & 1.9 \\
\hline Others & 57 & 4.3 & 12 & 4.4 & 69 & 4.3 \\
\hline
\end{tabular}

Then the association between Vitamin A supplementation status and illness history (fever, cough or rapid/difficulty in breathing, eye infection and diarrhea) was assessed using Multinomial Logistic Regression. Odds Ratio (OR) and Adjusted Odds Ratio (AOR) were computed accordingly.

The OR for all dependant variables was adjusted for intake and frequency of breastfeeding, and consumption of Vitamin A rich foods as appraised by 24 hours dietary recall method. In addition, the OR for fever was adjusted for sleeping under bed net the night before the survey, the OR for diarrhea was adjusted for type of usual water source and ownership of latrine, and the OR for eye infection was adjusted for ownership of latrine. However, history of Vitamin A supplementation was not significantly associated with all-cause morbidity $[\mathrm{AOR}=1.26 \quad(95 \% \mathrm{CI} \quad 0.95-1.67)]$, Fever $\quad[\mathrm{AOR}=1.26$ (95\%CI 0.89-1.77)], Cough or rapid breathing/difficulty in breathing $[\mathrm{AOR}=1.15 \quad(95 \% \mathrm{CI} \quad 0.77-1.72)]$, Eye infection $[\mathrm{AOR}=1.22$ (95\%CI 0.78-1.89)], and Diarrhea $[\mathrm{AOR}=0.98(95 \% \mathrm{CI}$ 0.64-1.52)].

\section{Discussion}

The current Vitamin A coverage (83.1\% among children 6-59 months and 13.2 among mothers in postpartum period) is significantly higher than the corresponding figure in $2005(\mathrm{P}<0.05)$. According to a similarly survey 
conducted by ESHE in 2005 in the area, the Vitamin A supplementation coverage was $74.0 \%$ among children aged 6-59 months, and $4.0 \%$ among mothers who had given birth in the preceding 12 month of the survey.

Despite this achievement, not more than one in seven women got the Vitamin A supplement during their postpartum period. This potentially increases risk of Vitamin A deficiency among lactating mothers and exclusively breastfed children.

Most of the Vitamin A supplementation coverage was attained through Enhanced Outreach Strategy (EOS) campaigns indicating the vitality of this service delivery outlet. However, since EOS is a temporary strategy for improving the coverage of supply constrained system and since it is highly dependant on foreign fund (28), sustainability of the coverage should get due consideration. The fact that very insignificant number of children got the supplement through sick child visit of health institutions and well child visit for growth monitoring, indicates the presence of missed opportunity for Vitamin A supplementation.

In this study, Vitamin A supplementation was not significantly associated with occurrence of diarrhea and symptoms of acute respiratory infection (cough or rapid breathing/ difficulty in breathing). A number of studies have documented similar findings. According to a double blind placebo controlled trial in Calcutta slum community by Biswas $\mathrm{R}$ et al. (20), Vitamin A supplementation was not significantly associated with the incidence of diarrheal episodes or ARI. Similarly, a meta-analysis of 9 randomized controlled trials by Itamar $\mathrm{G}$ et al. (29), indicated that vitamin A supplementation has no consistent protective effect on the incidence of diarrhea and ARI. Similar findings have also been reported by other studies (21-23).

In this study Vitamin A supplementation was not significantly associated with occurrence of eye infection. The association between Vitamin A supplementation and eye infection has not been studied deeply. However according to a randomized, double-blind, placebocontrolled trial by Filteau S et al. (30), Vitamin A supplementation status was not significantly associated either with epithelial integrity nor with measures of eye infection. Similarly, this study could not establish association between Vitamin A supplementation and occurrence of fever. A parallel finding has been reported in Ghana by Binka F et al. (31). According to this study, there was no difference in occurrence of fever and malaria parasitemia between children supplemented with vitamin $\mathrm{A}$ and those given placebo.

\section{Conclusions and Recommendations}

Significant improvement in Vitamin A coverage among children aged 6-59 months and women in the post partum period was witnessed. Among children aged 6-59 months, most of the vitamin A supplementation coverage was attained through Enhanced Outreach Strategy (EOS). Only insignificant number of children got the supplement through sick or well child visit of health institutions indicating the presence of missed opportunity. Although some studies demonstrated reduction of childhood illness with Vitamin A supplementation, this was not the case in the present study. The effect of Vitamin A supplementation on incidence of childhood illnesses should be assessed through longitudinal studies among Vitamin A deficient children.

\section{Limitations of the Study}

The findings of this study should be taken in consideration of the following limitations.

1. The study didn't use longitudinal design which is the ideal alternative to address such objectives.

2. The study can potentially underestimate the beneficial effect of vitamin A in reduction of childhood illnesses as it is not conducted exclusively among vitamin A deficient children.

\section{Acknowledgments}

The authors express their gratitude to JSI/ESHE for funding the study. Special thanks are also forwarded to all study subjects who volunteered in this study.

\section{Reference}

1. Colorado State University. Vitamin A, http://www.vivo.colostate.edu/hbooks/pathphys/ misctopics/ vitamina.html, Date Accessed Feb 28, 2008.

2. Linus Pauling Institute: Micronutriment Information: Vitamin A, http://lpi.oregonstate.edu/infocenter/ vitamins/vitaminA/, Date Accessed Feb 28, 2008.

3. John Hopkins Bloomberg School of Public Health. Tables on the Global Burden of Vitamin A Deficiency among Preschool Children and Women of Reproductive Age, http://www.jhsph.edu/chn/ globalVAD. html, Date Accessed May 09, 2009.

4. Kuhnlein HV, Pelto GH. Culture, Environment and Food to Prevent Vitamin A Deficiency, $1^{\text {st }}$ ed., Canada: IDRC/INFDC Publication, 1997.

5. WHO. Immunization Service Delivery and Accelerated Disease Control: Vitamin A, http://www.who.int/vaccines/en/vitamina.shtml, Date Accessed Mar 01, 2008.

6. FAO. FAO Report: Report on Nutritional Need of Children in Ethiopia, 1958.

7. Interdepartmental Committee on Nutrition for National Defense. Nutrition Survey in Ethiopia, 1959.

8. McLaren D. Age Specific Xerophthalmia Rates among Displaced Ethiopians, Arch Dis Child 1987; 62: 539-41.

9. West C, Wolde-Gebriel Z, Gebru H, Fisseha $T$. Severe Vitamin A Deficiency in a Rural Village in the Hararge Region of Ethiopia, Eur J Clin Nut 1993; 47: 104-14.

Ethiop. J. Health Dev. 2009;23(3) 
10. Tafesse Y, Fisseha T, Umeta M, Hidar J, Teka W. Vitamin A Deficiency: A Serious Threat in Dodota District in Central Ethiopia, East Afr Med J 1996; 73:303-7.

11. Haidar J, Demisse T, G/Sillasie HM, Fufa H, Biratu E. Vitamin A Deficiency Status in Tigray Region, Ethiopia, Ethiop J Health Dev 1999;13(2):87-91.

12. Tarik K, Olivier R, Timothy J, Margaret R. Prevalence of Vitamin A Deficiency in Children Aged 6-9 Years in Wukro, Northern Ethiopia, Bull World Health Organ 2001; 79:1-15.

13. ORC Macro and CSA Ethiopia. Ethiopian Demographic and Health Survey: DHS 2005, 2006.

14. Beaton G, Martorell R, L'Abbe K, Edmonton B, McCabe G, Ross A, et al. Effectiveness of Vitamin A Supplementation in the Control of Young Child Morbidity and Mortality in Developing Countries, Toronto: University of Toronto Press, 1992.

15. Glasziou P, Mackerras D. Vitamin A Supplementation in Infectious Diseases: a Metaanalysis, $B M J$ 1993;306: 366-70.

16. Fawiz W, ChaimersT, Herrera M, Mosteller F. Vitamin A Supplementation and Child Mortality: a Meta-analysis, JAMA 1993;269:898-903.

17. Victor M, Shawn K. Vitamin A Deficiency and Child Survival in Sub-Saharan Africa: A Reappraisal of Challenges and Opportunities, Food Nutr Bull 2005; 26; 348-54.

18. George H, Reynaldo M, Kristan A, Barry E, George M, Catharine R, et al. Vitamin A Supplementation and Child Morbidity and Mortality in Developing Countries, http://www.unu.edu/unupress/food/8f154e/8F154E0 4.htm, Date Accessed Feb 28, 2008.

19. Haidar J, Tsegaye D, Hailemariam D, Nekatibeb H, Muroki N. Vitamin A Supplementation on Child Morbidity, East Afr Med J 2003;80:17-21.

20. Biswas R, Biswas A, Manna B, Bhattacharya K, Dey R, Sarkar S. Vitamin A Supplementation and Childhood Morbidity from Diarrhea and Respiratory Infections: A Meta-analysis, J Pediatr 2003;142: 297-304.
21. Brown N, Roberts C. Vitamin A for Acute Respiratory Infection in Developing Countries: A Meta Analysis, Acta Peadiatrica 2007;93:1437-42.

22. Abdelijaber M, Monto A, Tilden R, Schork M, Tarwotjo I. The Impact of Vitamin A Supplementation on Morbidity: A Randomized Community Intervention Trial, Am J Public Health 1991;81:1654-6.

23. Humphrey J, Agoestina T, Usman A, Nurachim M, Subarda D, et al. Impact of Neonatal Vitamin A Supplementation on Infant Morbidity and Mortality, J Pediatr 1996;128:489-96.

24. Stansfield S, Pierre-Louis M, Lerebours G, Augustin A. Vitamin A Supplementation and Increased Prevalence of Childhood Diarrhea and Respiratory Infections, Lancet 1993;342:578-82.

25. Michael J, Tonny S, Chris L, Lawrence H. Vitamin A Supplementation Fails to Reduce Incidence of Acute Respiratory Illness and Diarrhea in PreschoolAge Indonesian Children, J Nutr 1997; 126: 434-7.

26. CSA Ethiopia. National Statistics of Ethiopia, 2005.

27. Wolayita Development Association. Profile of Wolayita Zone, http://www.wolaitta-da.org.et/ wolaitta. htm, Date Accessed Mar 1, 2009.

28. John L, Tesfaye C. The Cost of Child Health Days: A Case Study of Ethiopia's Enhanced Outreach Strategy (EOS), J Health Policy Planning 2008; 23:222-33.

29. Itamar G, Marc M, Michael G, Daniel M. Vitamin A Supplementation and Childhood Morbidity from Diarrhea and Respiratory Infections: A Metaanalysis, J Pediatr 2003;142:297-304.

30. Filteau S, Morris S, Tomkins A, et al. Lack of Association between Vitamin A Status and Measures of Conjunctival Epithelial Integrity in Young Children in Northern Ghana, European J Clin Nutr 1994;48:669-77.

31. Binka F, Ross D, Morris S, et al. Vitamin A Supplementation and Childhood Malaria in Northern Ghana, Am J Clin Nutr 1995;61:853-9. 